\title{
Gestión de marketing para el incremento de utilidades en la producción de conservas de pescado de la industria Don Martin S.A.C. Huacho, 2019.
}

\section{Marketing management for increase in profits in the production of fish conservations of industry don martin S.A.C. Huacho, 2019.}

\author{
Erickson Lorenzo Cruz Chero ${ }^{(1)}$; JoséMoisés Cueva Eusebio ${ }^{(2)}$; Cristhian Jesús Davila Yupanqui ${ }^{(3)}$; Jaime \\ Eduardo Gutiérrez Ascón ${ }^{(4)}$
}

\section{RESUMEN:}

Introducción: El objetivo principal de este proyecto fue: determinar la correcta Gestión de Marketing para el incremento de utilidades, en la producción de conservas de pescado de la industria Don Martin S.A.C. Huacho, 2019. Material y métodos: La investigación fue de tipo aplicada y de diseño cuasi experimental, tuvo un porcentaje de significancia del 5\% y nivel de confianza del $95 \%$. Se utilizó el software estadístico de Cristal Ball, para pronosticar las ventas de los próximos meses, y de esa manera elaborar las estrategias correspondientes en la gestión de Marketing. Resultados: La estacionalidad de las ventas de conserva de trozos de atún es de nivel 9 con desviación estándar 21,23 y SARIMA $(0,0,1)$ $(0,0,1)$ y U de Theil 0,4987 . Simulando 15000 veces, las ventas totales tiene Asimetría perfecta 0,00 y Curtosis 3,0. Al 95\% de nivel de confianza las ventas de atún se encuentran entre 3364 890, 45 a 4534 817,30 soles. Con las nuevas estrategias a implementar se espera lograr un incremento en las ventas de un $10 \%$. Discusión: Se confirma que, con un correcto análisis de la producción y su efecto en las utilidades, se puede priorizar el producto al cual se le debe poner mayor atención.

Palabras Clave: Marketing, Utilidades, Cristal Ball, Pronosticar.

\begin{abstract}
:
Introduction: The main objective of this project was: to determine the correct Marketing Management for the increase of profits, in the production of canned fish of the industry Don Martin S.A.C. Huacho, 2019. Material and methods: The research was of the applied type and of a quasi-experimental design, had a significance percentage of 5\% and a confidence level of $95 \%$. The Crystal Ball statistical software was used to forecast the sales of the next months, and in this way develop the corresponding strategies in Marketing management. Results: The seasonality of sales of canned tuna chunks is level 9 with standard deviation 21.23 and SARIMA (0.0.1) (0.0.1) and $U$ of Theil 0.4987. Simulating 15,000 times, total sales have Perfect Asymmetry 0.00 and Curtosis 3.0. At 95\% confidence level tuna sales are between 3364 890, 45 to 4534817.30 soles. With the new strategies to be implemented, a $10 \%$ increase in sales is expected. Discussion: It is confirmed that, with a correct analysis of production and its effect on profits, the product to which greater attention should be given can be prioritized.

Keywords: Marketing, Utilities, Crystal ball, Forecast.

\footnotetext{
${ }^{1}$ Autor corresponsal Universidad Nacional José Faustino Sánchez Carrión. Huacho. Perú. . Estudiante VI ciclo. Facultad de Ingeniería Industrial Sistema e Informática. Email: 0331121001 @unjfsc.edu.pe. Teléfono 973193819 http://orcid.org/0000-0002-4856-381X

2 . Universidad Nacional José Faustino Sánchez Carrión. Huacho. Perú. Estudiante VI ciclo. Facultad de Ingeniería Industrial Sistema e Informática http://orcid.org/0000-0002-7570-004X

3 . Universidad Nacional José Faustino Sánchez Carrión. Huacho. Perú. Estudiante VI ciclo. Facultad de Ingeniería Industrial Sistema e Informática http://orcid.org/0000-0002-2226-5494

4 . Universidad Nacional José Faustino Sánchez Carrión. Huacho. Perú. Ingeniero. Facultad de Ingeniería Industrial Sistema e Informática https://orcid.org/0000-0003-4065-3359
} 


\section{INTRODUCCIÓN}

El presente proyecto "Gestión de marketing para el incremento de utilidades, en la producción de conservas de pescado de la Industria Don Martin S.A.C. Huacho, 2019.”, se realizódebido a las problemáticas encontradasen laempresa.Unavezque seidentificó dichos problemasnoscentramos en uno de ellos, en este caso nos enfocaremos en las ventas y así poder saber cuántas utilidades obtiene la empresa por cada temporada.

Las ventas poco significativas han hecho que pongamos nuestra atención en la realización de este proyecto de investigación, para lograr incrementar las ventas en la empresa propusimos una adecuada gestión del marketing.

ParalocualserealizarápronósticosmedianteelprogramaCristall Ballparalassiguientes temporadas de dos trimestres de junio a noviembre, se trabajará en base a datos históricos de los años 2016, 2017, 2018 y parte del 2019, se evaluarán los datos obtenidos mediante distintos gráficos para poder obtener una decisión más eficiente y certera.

Por lo tanto nuestro objetivo principal es desarrollar la Gestión del Marketing para incrementar las utilidades en el área de producción de conservas de Pescado de la industria Don Martin S.A.C. Huacho, 2019, actualmente el marketing se constituye como una poderosa herramienta que utilizada de manera correcta y eficiente ayuda a incrementar de manera considerable las utilidades de un negocio, tiene su aplicación a cualquier tipo de empresa y en la actualidad el marketing digital se da con más impacto debido al internet.

\section{MATERIALES Y MÉTODOS}

La investigación fue de tipo aplicada y analítica, los datos que utilizamos corresponden a las ventas realizadas por la Industria Don Martin S.A.C., en los meses correspondientes a enero, febrero, marzo, abril y mayo. El software que se empleó para pronosticar las ventas que se realizaran en los próximos 2 trimestres de producción y ventas fue Oracle Crystal Ball, exactamente con su herramienta Predictor, esta nos permite predecir el comportamiento de una muestra determinada. La utilización de esta herramienta es muy fácil solo se le tiene que realizar unos ajustes antes de realizar la predicción, por ejemplo, se tiene que ver las unidades con las que estamos trabajando de la misma manera se debe determinar si los datos son estaciones o no, para de esta manera elegir el mejor método y no tener contradicciones en la predicción que queremos hacer. Con este software también se puede determinar cuál es el producto que tiene mayor influencia en el registro de ventas y por consiguiente también su importancia en la generación de utilidades. Pero todo esto no hubiera sido posible sin la correcta utilización de nuestra principal herramienta que es Microsoft Excel, recalcamos que Oracle Crystal Ball es una extensión de este programa, es decir si no tenemos el software de Excel, no se podrías utilizar las herramientas de Crystal Ball, y por consiguiente el proyecto no se hubiera podido llevar a cabo, de ahí que resaltamos su importancia.

En la tabla 1 se muestran las variables dependiente e independiente, así como sus dimensiones e indicadores correspondientes 
Tabla 1.

Matriz de Operacionalización de las variables

GESTIÓN DE MARKETING PARA EL INCREMENTO DE UTILIDADES EN LA PRODUCCIÓN DE CONSERVAS DE PESCADO DE LA INDUSTRIA DON MARTIN S.A.C. HUACHO, 2019.

\begin{tabular}{|c|c|c|c|c|}
\hline VARIABLES & Definicion conceptual & Definicion operacional & Dimensiones & Indicadores \\
\hline \multirow{3}{*}{ 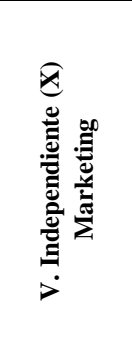 } & \multirow{3}{*}{$\begin{array}{l}\text { Marketing:Es el proceso de } \\
\text { planificación y ejecucion del } \\
\text { concepto, precio, promoción y } \\
\text { distribución de ideas, bienes y } \\
\text { servicios para crear intercambios } \\
\text { que satisfagan objetivos individuales } \\
\text { y colectivos.William } \\
\text { Stanton.Fundamentos de } \\
\text { marketing.(1964). } \\
\text { ISBN 978-970-10-6201-9 }\end{array}$} & \multirow{3}{*}{$\begin{array}{l}\text { Marketing: Es el resultado de } \\
\text { las actividades propias de la } \\
\text { gestión comercial que se logra } \\
\text { debido al manejo del estudio de } \\
\text { mercado y de la Capacitación en } \\
\text { ventas; que, tienen como fin el } \\
\text { logro de los objetivos } \\
\text { empresariales. } \\
\text { Cruz et al. (2019) }\end{array}$} & D1: Gestión comercial & $\begin{array}{l}\text { * Satisfacción del cliente. } \\
\text { * Expansión del mercado. }\end{array}$ \\
\hline & & & D2: Estudio de mercado & $\begin{array}{l}* \text { Preferencias } \\
\text { * Demografía }\end{array}$ \\
\hline & & & $\begin{array}{l}\text { D3: Capacitación en } \\
\text { ventas }\end{array}$ & $\begin{array}{l}* \text { Habilidades } \\
* \text { Recursos }\end{array}$ \\
\hline \multirow{2}{*}{ 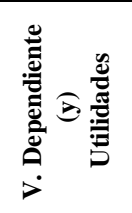 } & \multirow{2}{*}{$\begin{array}{l}\text { Utilidades.Utilidad que resulta ser } \\
\text { una propiedad física de los bienes, } \\
\text { independiente de todo aspecto } \\
\text { cuantitativo. Baltra. Estudio } \\
\text { económico. (1973) } \\
\text { ISBN: } 1-08-84-2-1\end{array}$} & \multirow{2}{*}{$\begin{array}{l}\text { Utilidades. Es el producto } \\
\text { de la restabilidad en una } \\
\text { empresa una vez alcanzada } \\
\text { la competitividad en el } \\
\text { mercado. Cruz et al. (2019) }\end{array}$} & d1:Rentabilidad & $\begin{array}{l}* \text { Precios } \\
* \text { Ventas }\end{array}$ \\
\hline & & & d2: Competitividad & $\begin{array}{l}* \text { Precio } \\
* \text { Calidad }\end{array}$ \\
\hline
\end{tabular}

\section{Variable Independiente: Marketing}

El Marketing se encarga de estudiar el comportamiento de los mercados y de las necesidades de los consumidores. Además, analiza la gestión comercial de las compañías con la finalidad de atraer, captar, retener y fidelizar a los clientes finales a través de la satisfacción de sus deseos y resolución de sus problemas.

Philip Kotler y Gary Armstrong (2007), afirma que "Sería difícil imaginar un mundo sin marketing. Pero puede ser igualmente difícil apreciar la importancia que el marketing real desempeña en la mayoría de los aspectos de nuestra vida. Como cosa hecha que siempre está ahí, solemos subestimar los medios apoyados en gran medida por la publicidad, el gran surtido de bienes distribuidos por tiendas cercanas a nuestros hogares o la facilidad con que podemos hacer compras. Para apreciar mejor, consideremos por un momento cómo desempeña el marketing una de las funciones principales en la economía global, en el sistema socioeconómico de cualquier país, en cualquier organización y en la vida de usted" p.18

\section{Variable dependiente: Utilidades}

Las utilidades es lo que la empresa gana en un periodo determinado que por lo general es de un año.

David E. Stout (2013), afirma que "La utilidad es el beneficio económico efectivo que obtienen los dueños de una empresa u organización. En ella se consideran no solo los ingresos obtenidos sino también todos los gastos en los que fue necesario incurrir para conseguirlos" p. 218

En la tabla 2 se muestran los datos históricos en ventas de las tres líneas de producción más representativas de la Industria Don Martin S.A.C., los datos recolectados abarcan desde el 2016 hasta el mes de mayo del presente año 2019, 
Tabla 2: Demanda por línea de producción de la empresa

\begin{tabular}{|c|c|c|c|c|}
\hline Año & Fecha & $\begin{array}{l}\text { Ventas de } \\
\text { conserva de atún } \\
\text { en trozos } \\
\quad(\text { Cajas })\end{array}$ & $\begin{array}{l}\text { Ventas de } \\
\text { conserva de filete } \\
\text { de atún } \\
\quad \text { (Cajas) }\end{array}$ & $\begin{array}{l}\text { Ventas de } \\
\text { conserva de } \\
\text { jurel en trozos } \\
\quad \text { (Cajas) }\end{array}$ \\
\hline \multirow{12}{*}{2016} & enero & 599 & 124 & 95 \\
\hline & febrero & 561 & 119 & 70 \\
\hline & marzo & 581 & 115 & 146 \\
\hline & abril & 599 & 122 & 89 \\
\hline & mayo & 561 & 117 & 125 \\
\hline & junio & 582 & 120 & 93 \\
\hline & julio & 563 & 112 & 70 \\
\hline & agosto & 550 & 123 & 137 \\
\hline & septiembre & 537 & 128 & 100 \\
\hline & octubre & 597 & 131 & 121 \\
\hline & noviembre & 532 & 113 & 70 \\
\hline & diciembre & 575 & 130 & 95 \\
\hline \multirow{12}{*}{2017} & enero & 562 & 118 & 134 \\
\hline & febrero & 608 & 154 & 109 \\
\hline & marzo & 607 & 147 & 78 \\
\hline & abril & 577 & 139 & 69 \\
\hline & mayo & 572 & 151 & 87 \\
\hline & junio & 555 & 117 & 104 \\
\hline & julio & 572 & 146 & 115 \\
\hline & agosto & 557 & 138 & 75 \\
\hline & septiembre & 594 & 153 & 90 \\
\hline & octubre & 560 & 149 & 77 \\
\hline & noviembre & 602 & 151 & 68 \\
\hline & diciembre & 594 & 133 & 83 \\
\hline \multirow{12}{*}{2018} & enero & 553 & 154 & 114 \\
\hline & febrero & 600 & 126 & 77 \\
\hline & marzo & 564 & 120 & 65 \\
\hline & abril & 548 & 130 & 100 \\
\hline & mayo & 599 & 143 & 124 \\
\hline & junio & 585 & 123 & 60 \\
\hline & julio & 547 & 137 & 115 \\
\hline & agosto & 608 & 116 & 113 \\
\hline & septiembre & 564 & 140 & 119 \\
\hline & octubre & 580 & 111 & 99 \\
\hline & noviembre & 608 & 135 & 121 \\
\hline & diciembre & 578 & 152 & 136 \\
\hline \multirow{11}{*}{2019} & enero & 562 & 137 & 67 \\
\hline & febrero & 550 & 126 & 62 \\
\hline & marzo & 550 & 117 & 89 \\
\hline & abril & 554 & 105 & 97 \\
\hline & mayo & 605 & 145 & 79 \\
\hline & junio & & & \\
\hline & julio & & & \\
\hline & agosto & & & \\
\hline & septiembre & & & \\
\hline & octubre & & & \\
\hline & noviembre & & & \\
\hline
\end{tabular}


En la Figura 1 se muestran si nuestros datos poseen estacionalidad o no, además de mostrarse otras características que poseen lo datos históricos recolectados

La estacionalidad o variación estacional de una serie temporal es la variación periódica y predecible de la misma con un periodo inferior o igual a un año, a raíz de poder ver si nuestros datos son estacionales o no podemos elegir el mejor método con el que nuestra predicción se realizará.

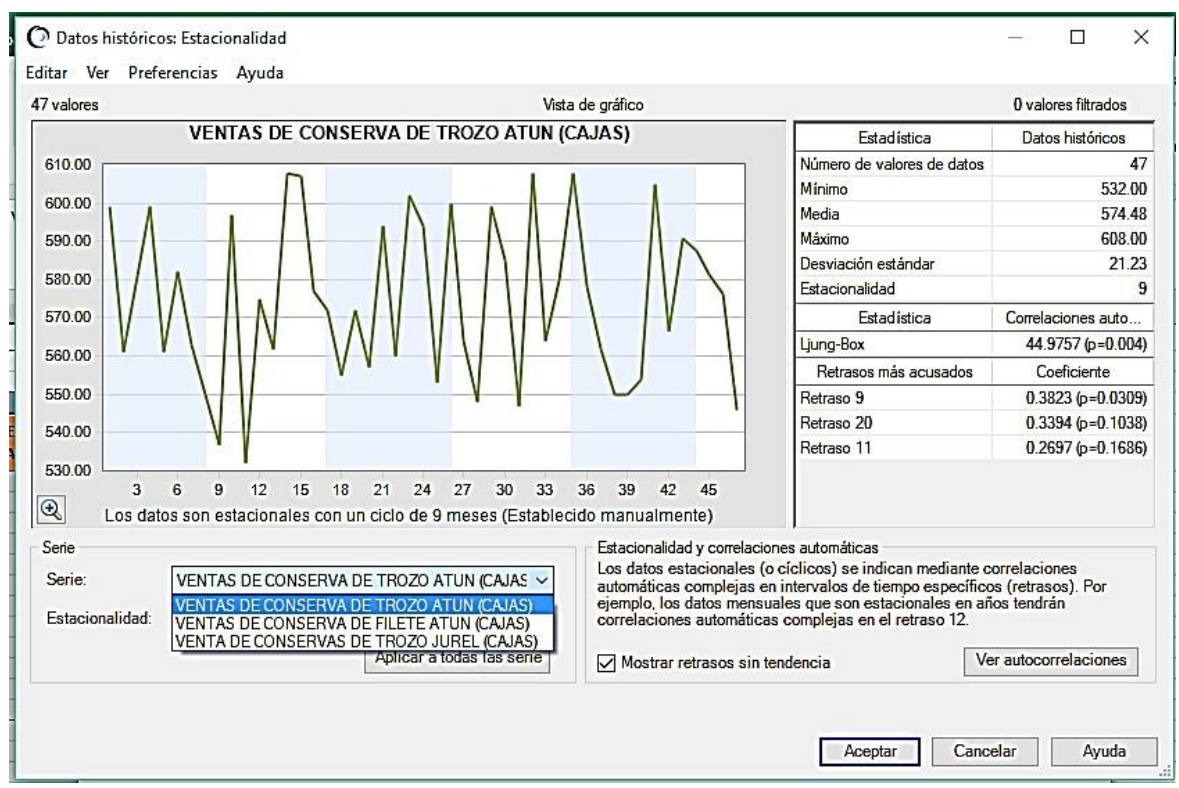

Fig. 1: Determinación de la estacionalidad y otras características de los datos

En la Figura 2 se va a seleccionar el método con el que queremos trabajar para realizar la predicción, nuestras tres columnas de datos muestran un comportamiento estacional por lo que solo dejamos seleccionado el método estacional y el método de ARIMA

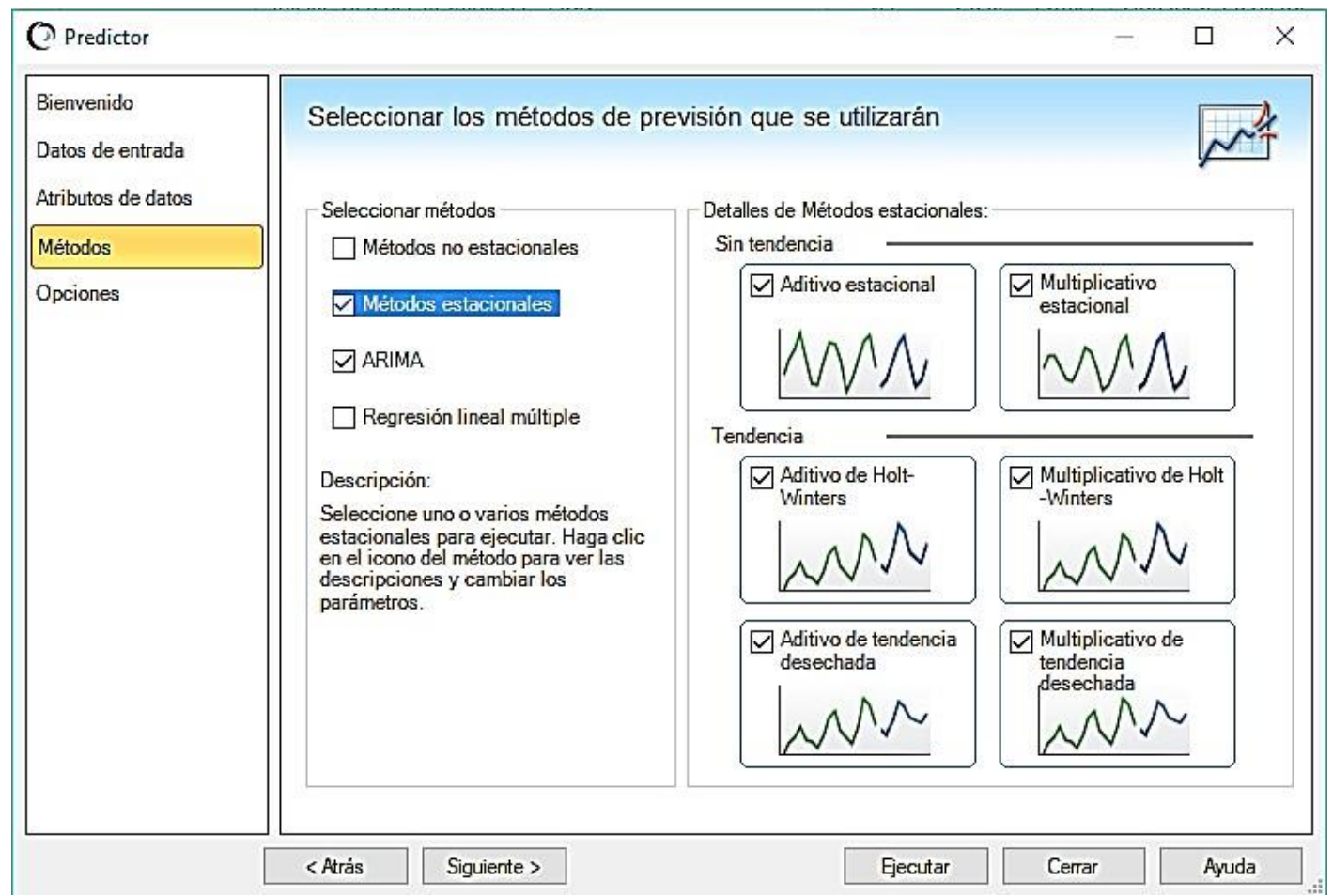

Fig. 2: Selección de los métodos con el cual se realizará la predicción 
En la figura 3 se muestra el comportamiento de la predicción echa para las ventas por cajas de los próximos 2 trimestres de las conservas de trozo de atún

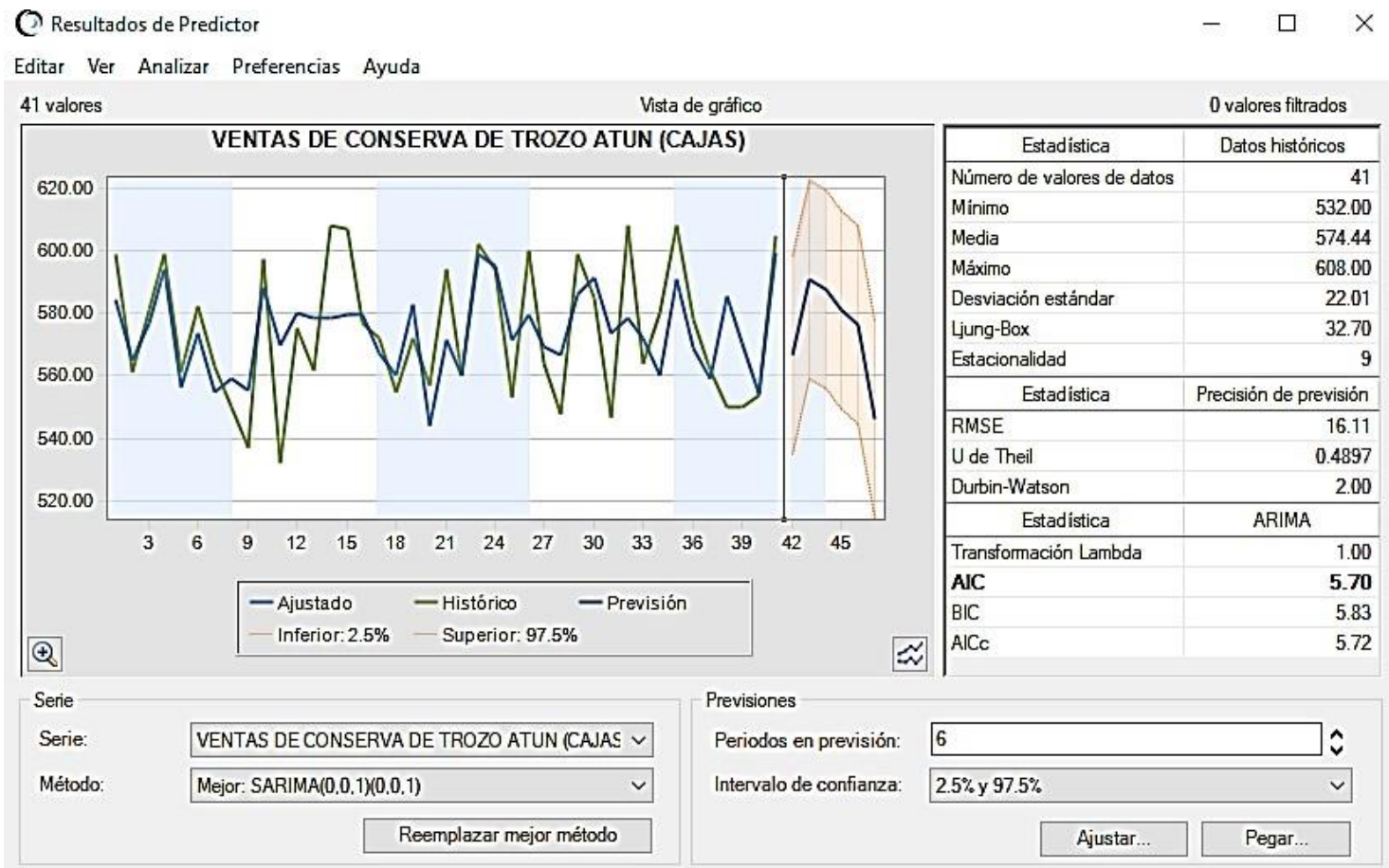

Fig. 3: Características de la predicción para las conservas de trozo de atún.

En la figura 4 se muestra el comportamiento de la predicción echa para las ventas por cajas de los próximos 2 trimestres de las conservas de filete de atún.

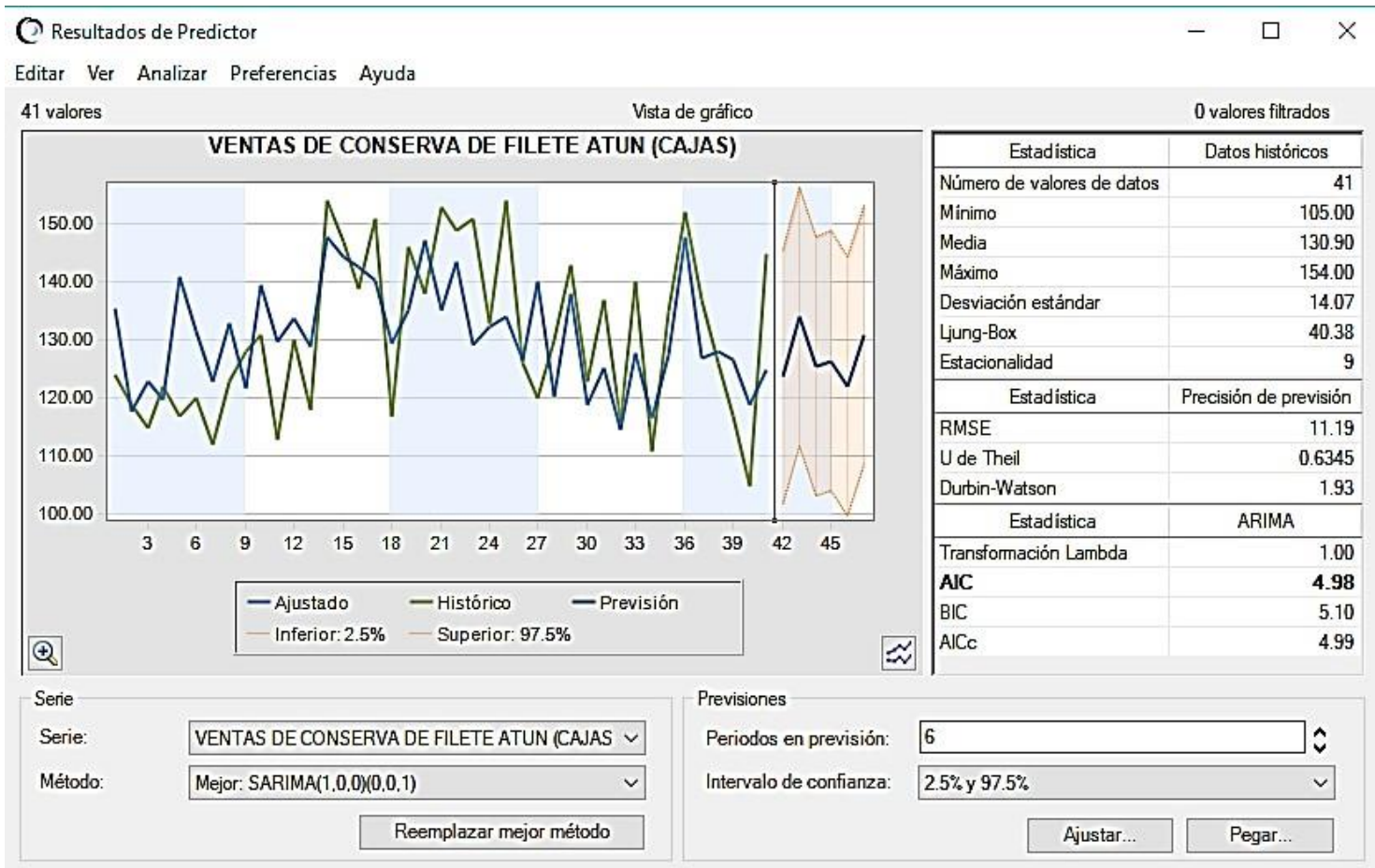

Fig. 4: Características de la predicción para las conservas de filete de atún. 
En la figura 5 se muestra el comportamiento de la predicción echa para las ventas por cajas de los próximos 2 trimestres de las conservas de trozo de jurel.

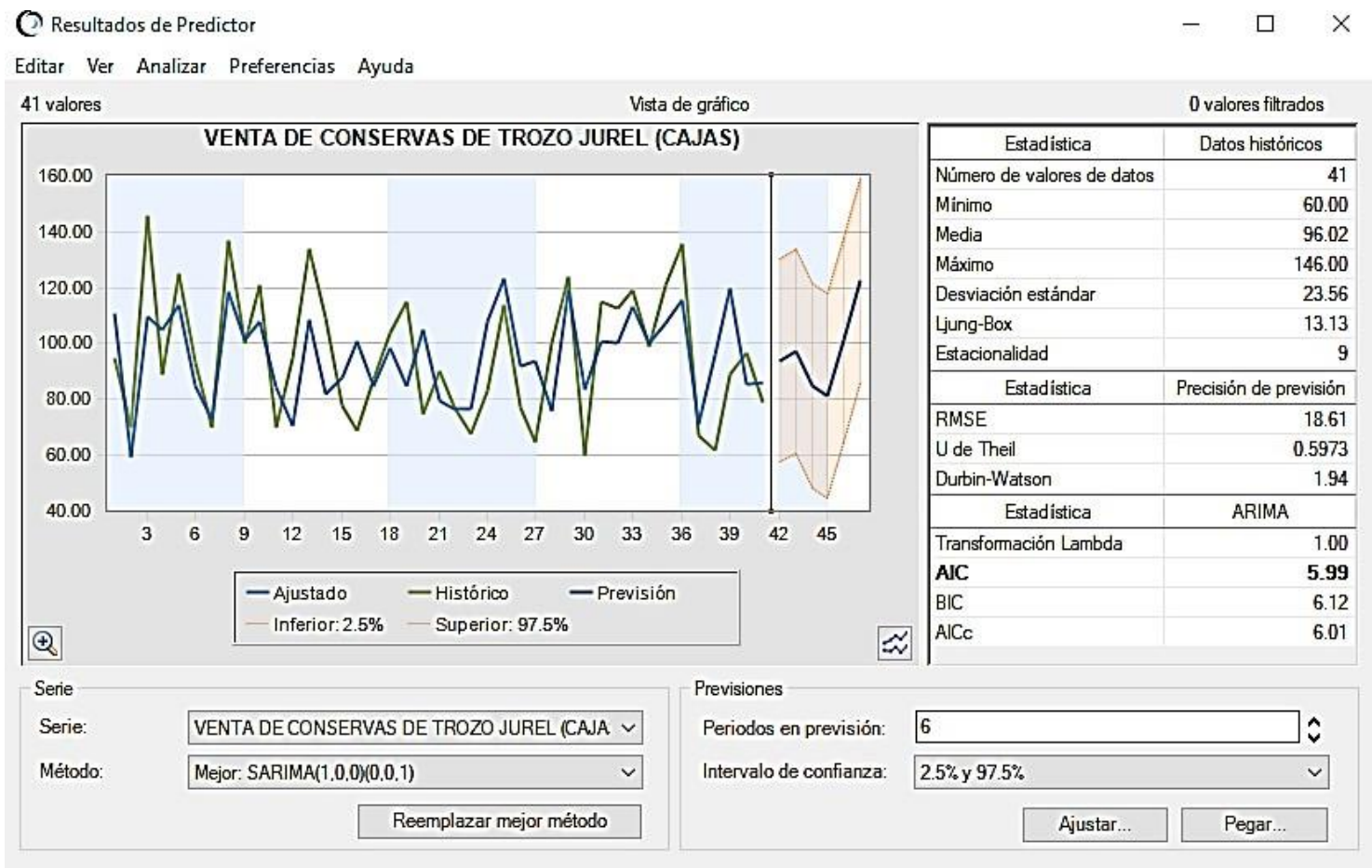

Fig. 5: Características de la predicción para las conservas del trozo de Jurel.

\section{RESULTADOS}

En la tabla 3 ya se ven reflejados los valores del comportamiento de ventas obtenidas con la herramienta Predictor de Crystal ball, para cada una de las líneas de producción más representativas de la industria Don Martin S.A.C.

Tabla 3.

Resultados de la predicción

\begin{tabular}{c|l|c|c|c}
\hline Año & Fecha & $\begin{array}{l}\text { Ventas de } \\
\text { conserva de } \\
\text { atún en trozos } \\
\text { (Cajas) }\end{array}$ & $\begin{array}{l}\text { Ventas de } \\
\text { conserva de } \\
\text { filete de atún } \\
\text { (Cajas) }\end{array}$ & $\begin{array}{l}\text { Ventas de } \\
\text { conserva de } \\
\text { jurel en trozos } \\
\text { (Cajas) }\end{array}$ \\
\hline \multirow{5}{*}{$\mathbf{2 0 1 9}$} & enero & 562 & 137 & 67 \\
& febrero & 550 & 126 & 62 \\
& marzo & 550 & 117 & 89 \\
& abril & 554 & 105 & 97 \\
& mayo & 605 & 145 & 79 \\
& junio & $\mathbf{5 6 7}$ & $\mathbf{1 2 4}$ & $\mathbf{9 4}$ \\
& julio & $\mathbf{5 9 1}$ & $\mathbf{1 3 4}$ & $\mathbf{9 7}$ \\
& agosto & $\mathbf{5 8 8}$ & $\mathbf{1 2 6}$ & $\mathbf{8 5}$ \\
& septiembre & $\mathbf{5 8 1}$ & $\mathbf{1 2 7}$ & $\mathbf{8 1}$ \\
& octubre & $\mathbf{5 7 6}$ & $\mathbf{1 2 2}$ & $\mathbf{1 0 2}$ \\
& noviembre & $\mathbf{5 4 6}$ & $\mathbf{1 3 1}$ & $\mathbf{1 2 3}$ \\
\hline
\end{tabular}

En la Fig. 6 se muestra la distribución del comportamiento de nuestras ventas, con un nivel de significancia del $5 \%$ y un nivel de confianza del $95 \%$. Se afirma que las ventas están entre los 
S/.3364890.45 y S/.4534817.30, además presenta una curtosis de tipo leptocúrtica de 3.00, lo que nos dice que presenta un alto nivel de ganancia o un alto nivel de pérdida.

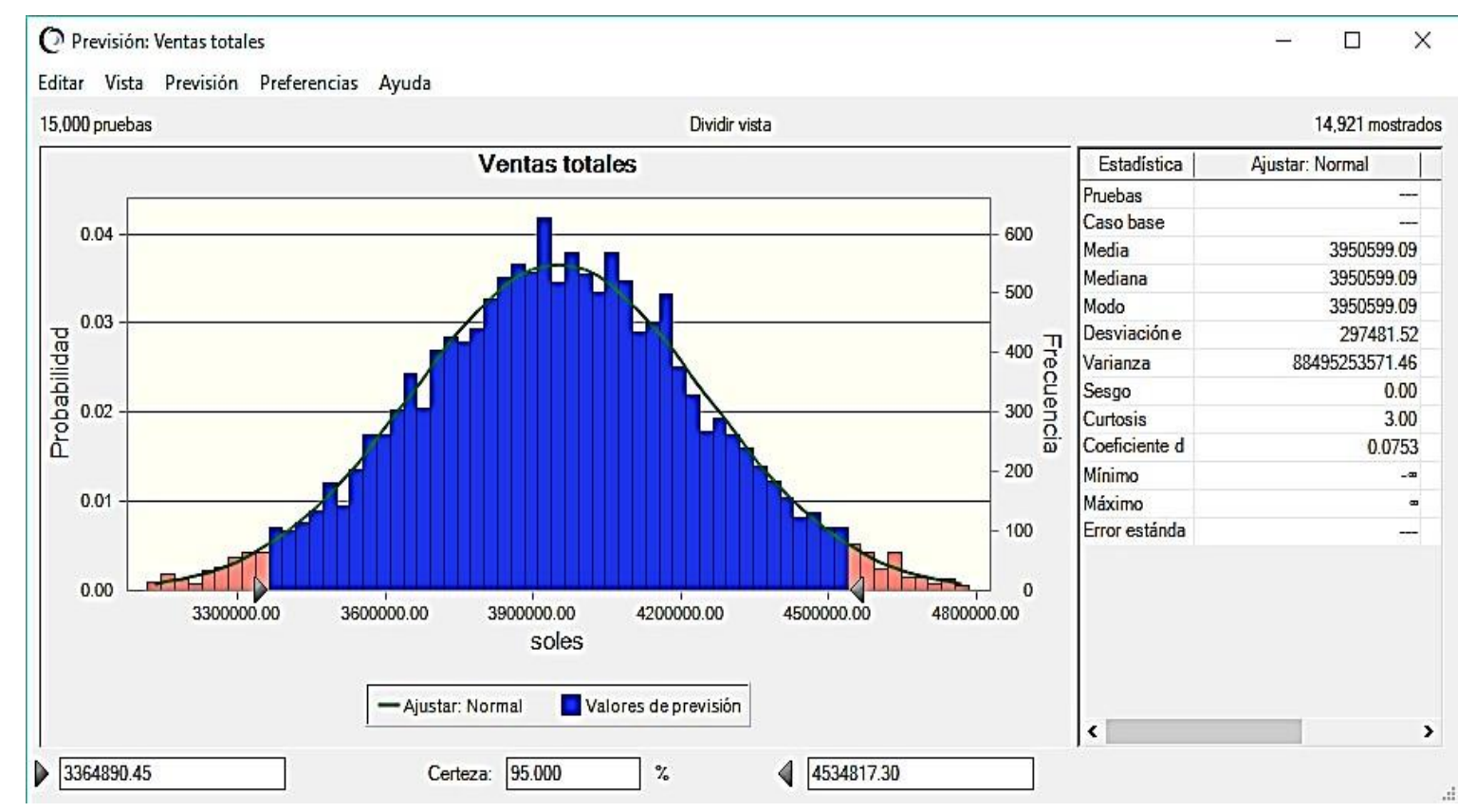

Fig. 6: Probabilidad de Ventas

En la Fig. 7 se muestran los resultados luego de haber utilizado la herramienta de análisis tornado, según estos datos obtenidos las ventas que Influyen de manera significativa en los ingresos corresponde al producto de conserva de trozo de atún, con esto concluimos, que este producto es el más importante para las ventas de la empresa.

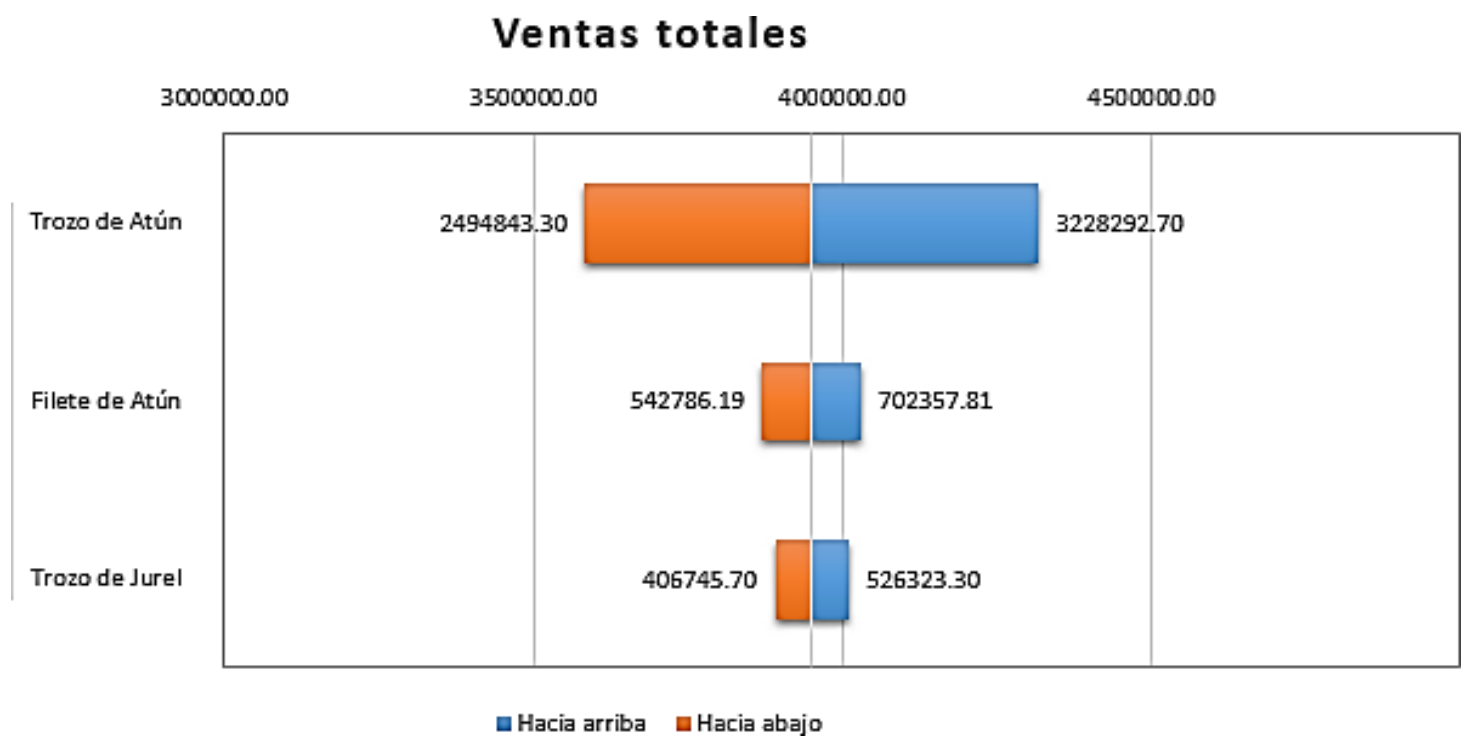

Fig. 7: Gráfico del Análisis de Tornado 
En la Fig. 8 de la misma manera se muestran los resultados luego de haber utilizado la herramienta de análisis Spider, este análisis también nos indica que el producto de mayor importancia para la empresa es el trozo de atún, el comportamiento de las ventas del trozo de atún es directamente proporcional a los ingresos generados, de tal manera que si las ventas de la conserva de trozo de atún aumentan o disminuyen los ingresos se verán afectados directamente de manera significativa.

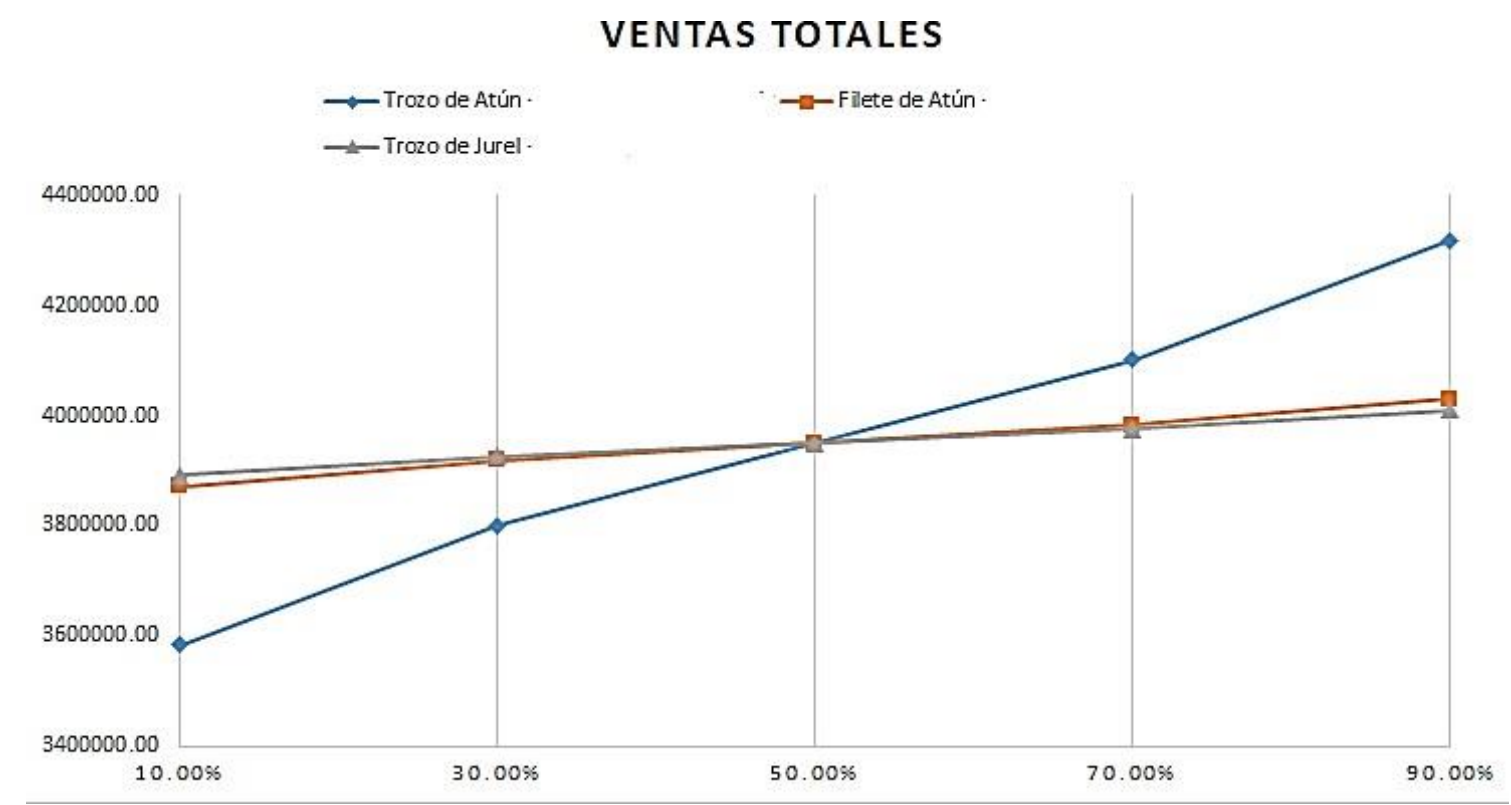

Fig. 8: Gráfico del análisis de Spider

\section{DISCUSION}

Luego de Realizar la predicción para las ventas de los próximos 2 trimestres del año 2019, también realizamos el análisis estadístico con los dos métodos que a nuestro parecer son los más representativos, estos son el método de tornado y el método de Spider. Estos dos métodos no indican que el producto más importante al cual debemos de ponerle una mayor atención es la conserva de trozos de atún, esta tiene un mayor impacto en los ingresos por ventas a comparación con los demás productos.

Gracias a estos resultados ya se pueden ir definiendo las estrategias de Marketing correspondientes, para lograr un incremento en las ventas, enfocándonos por mantener y/o elevar las ventas de la conserva de trozo de atún, de la misma manera aplicar una estrategia de marketing adecuada para lograr incrementar las ventas de los otros productos que no son tan influyentes.

\section{REFERENCIAS}

Bach. Orozco Cardozo Eduard Saúl (2015). Plan de mejora para aumentar la productividad en el área de producción de la empresa confecciones deportivas todo sport. Trabajo de Titulación. Chiclayo, Perú

Stefanie Johanna de Freitas Guzmán (2017). propuesta de marketing con el fin de incrementar las ventas en la empresa arrendauto s.a. Trabajo de Titulacion. Guayaquil, Colombia.

Asanza Guapacasa Wendy Elizabeth, Cheme Pilco Estefany Cinthya, Yánez Delgado Jorge Alberto (2013). Plan de ventas para optimizar la comercialización en la empresa Equiservisa s.a. Trabajo de Titulación. Guayaquil, Colombia.

Edward J. Blocher, David E. Stout, Gary Cokins, Kung H. Chen. Administración de costos. México: Editorial McGraw-Hill 
Vera Castro, Jary Jim (2016). Aplicación del sistema costos por órdenes de trabajo y su incidencia en la rentabilidad de la empresa industrial de poliestireno, nexpol s.a.c.

Trabajo de titulación. Lima, Perú.

William J. Stanton,Michael J. Etzel, Bruce J. Walker (1964). Fundamentos del Marketing. México, Editorial McGraw-Hill 